encouragement, and has become in many instances the central focus of the district. The clinical pathologist has often become the key consultant of the community to general practitioner and consultant alike; an artist with the needle (and what a mess a clumsy venepuncture or lumbar puncture can be); knowledgeable about modern advances and with a laboratory which, like its larger counterpart in teaching hospitals, is usually a meeting-place for intellectual medical discussion. Here, over the inevitable cup of tea, the medical men of the district come to discuss their problems, to seek advice, and to keep themselves up to date.

A landmark in the evolution of clinical pathology in this country was the foundation of the Association of Clinical Pathologists in 1927. There are similar organizations in the United States and in other countries, whilst the formation of a European Association was completed in 1947. In England, the association was started by a group of men drawn largely: from the non-teaching hospitals of the Provinces, who desired to have a society where there was emphasis on the practical aspects of pathology as applied to the bedside. The association is now one of the most flourishing in the country and publishes-in co-operation with the British Medical Association -its own journal.

The further development of the fascinating subject of clinical pathology will be an important aspect of the National Health Service. Though some of this work, especially bacteriology and histology, can be done in Regional laboratories in an impersonal manner, there will always be a far larger amount which necessitates the bringing of the patient to the pathologist or the pathologist to the patient. This, in places where distances are large and the population scattered, presents many problems. In this country, happily, the evolution of clinical pathology has not led to an attitude of mind where the patient becomes a mere number attached to a wad of routine laboratory reports among which, with a fine disregard for economic factors and intelligent selection, one result may occasionally be found which supplies a diagnostic finding. Fortunately, those who have practised the subject have been at pains to see that the personal side has prevailed, so that a clinical examination has orientated the laboratory work and the patient has remained a human problem. It is to be hoped that nationalization will not alter this typically British tradition, but there is inevitably a danger that bureaucracy may insidiously submerge humanitarianism and come to regard all clinico-pathological problems in terms of the appropriate Government form. Should this come to pass, then the fine traditions of the past half-century will disappear for ever.

My thanks are due to Dr. E. Ashworth Underwood, of the Wellcome Historical Medical Museum, and Mr. Leslie T. Morton, of the staff of the British Medical Journal, for their help in preparing this article.

Speech as a Septic Influence.--" Professor Flügge . . . might quote the same text in support of the doctrine which he preaches that speech is a means of distributing disease germs. He has shown that from the mouth of a person who is speaking come bacteria contained in little bubbles of saliva, which after remaining suspended some time in the air are scattered through the surrounding atmosphere. ... One may conjure up a prophetic vision of the twentieth-century surgeon with antiseptic mask, beard-bag, gloves, and sterilized robe, operating within a glass sanctuary into which no one is admitted except after the fullest disinfectant lustration." From an annotation in the British Medical Journal of February 17, 1900 (p. 401).

\section{PREVENTION AND CONTROL OF INFECTION}

\author{
BY
}

ROBERT CRUICKSHANK, M.D., F.R.C.P., D.P.H. Professor of Bacteriology, St. Mary's Hospital Medical School

A few years ago C.-E. A. Winslow, the doyen of preventive medicine in America, wrote a book called The Conquest of Epidemic Disease. Although the title sounded rather pretentious and euphemistic, Winslow's object was to trace the development of epidemiological concepts about the causation of epidemic disease from the time of the biblical belief in visitations from heaven or hell, through the theories on spontaneous generation and miasms, to the present-day proof of the transmissible germ; to show, in fact, that, since the aetiology and modes of spread of most epidemic diseases were now known, the means for their control should be available.

Beginning. with the acute observations of Hippocrates in his Airs, Waters, and Places on the epidemiology of such conditions as the ague, dysentery, and chest infections, the road to knowledge of the causation of infectious diseases has been slow and tortuous, and was blocked for a thousand years during the "Dark Ages." The pandemic of plague in the fourteenth century, which was so obviously contagious, was followed by a pandemic of syphilis about A.D. 1500 which allowed Fracastorius to elaborate his clear and convincing principles on the spread of disease by contagion, although he conceived the transmissible agent as being gaseous rather than living particulate matter. In spite of Kircher's scientific arguments in favour of the "contagium animatum" and Leeuwenhoek's microscopical demonstration of the little animalcules in the seventeenth century, the germ theory was again stayed during the eighteenth century, largely by Sydenham's advocacy of the "Epidemic Constitution," and it was not until the latter half of the nineteenth century that the genius of Pasteur and the painstaking researches of Koch firmly established the microbial aetiology of infection. By 1900 the heyday of bacteriological discovery had passed, for the causal organisms of most major pestilences-typhoid, dysentery, cholera and plague, tuberculosis, pneumonia and diphtheria, meningitis, and gonorrhoea-had been identified.

But there still remained many puzzles. The aetiological agents of typhus, syphilis, yellow fever, scarlet fever, and whooping-cough were still undetermined, while the occurrence of infection among those who had no obvious contact with the sick, and, per contra, the failure of others to develop infection in spite of such close contact, were still unexplained phenomena. The importance of the carrier in the spread of infection, the principles of immunology, and the factors determining the virulence of an organism are developments of the past half-century. At the beginning of the twentieth century there was still a great deal of spade-work to be done in the methods of isolation and identification of specific pathogens, so that opportunities for devising new microbiological techniques abounded. The typhoid bacillus was separated from the colon bacillus by fermentation reactions, the bovine type of tubercle bacillus was differentiated from the human type, and the botanical classification of bacteria as agents of infection became an absorbing study for the rank and file of the new race of bacteriologists who were still closely attached and subservient to the morbid anatomists. However, men of ideas like Ehrlich, Bordet, Almroth Wright, and Theobald 
Smith were not wanting, and they were turning their attention to the many problems in the control of infectious disease ; searching for the reservoirs of the infecting organism : tracing its mode of spread to new susceptible hosts ; studying new methods for protecting the host against infection ; or attempting to cure the infection by some specific therapy. Progress in the fight against infection during the past fifty years may best be reviewed under each of these four headings.

Progress in this field is often represented as being strikingly successful because of the rapid decline in the death rates from some of the common infectious diseases -for example, scarlet fever, measles, and whooping-cough. It is, however, doubtful if this reduction in mortality has owed much to specific measures aimed at the control and prevention of these infections, since there has been no corresponding decline in morbidity. A general improvement in social conditions, including nutrition, has probably been the main contributor to the reduction in case mortality until the new chemotherapy lent a helping hand. The case is otherwise, of course, with the intestinal infections, where there has been a great reduction in both morbidity and mortality rates.

\section{The Reservoirs of Infection}

The careful observations and deductive reasoning of John Snow and William Budd in the middle of the nineteenth century had established in pre-Pasteurian days the main facts about the spread of cholera and typhoid fever, but it frequently happened that outbreaks of these infections could not be traced to a primary case of the disease. In his studies on cholera Koch showed that convalescent cases or contacts with minor symptoms were excreting the vibrio, and these carriers could therefore explain the spread of infection by apparently healthy persons. This concept of the symptomless carrier as a link in the chain of infection was soon proved to be applicable to other infections. Koch and his associates, using the new Drigalski-Conradi culture medium, were able to demonstrate the presence of 72 missed cases or carriers of typhoid fever in a group of villages where only eight clinical cases had been reported. About the same time Park and Beebe demonstrated the occurrence of convalescent throat-carriers of the diphtheria bacillus, while later the meningococcus was found in the throats of convalescent cases and symptomless contacts, and dysentery bacilli in the stools of both contact and convalescent cases. The epidemiological importance of the carrier gradually became recognized, and in 1912 a monograph was published on "The Carrier Problem in Infectious Disease" by Ledingham and Arkwright, which, with its wealth of information, further helped to focus attention on this important reservoir of infection.

It became established that the gall-bladder was the main breeding-ground of the bacillus in the chronic typhoid carrier, and that this condition was four times more common in women than in men, particularly in the middle-aged matron who was also prone to gall-stones. In other words, a diseased organ or tissue was apparently a prerequisite of the persistent carrier condition. Similar findings emerged in the case of the diphtheria carrier, who usually was shown to have diseased tonsils or unhealthy nasal mucous membranes.

The evidence that the infecting organism persists in the tissues during convalescence in a steadily decreasing proportion of cases, which was first demonstrated for diphtheria and typhoid, has been extended to other bacterial and virus infections as techniques for the isolation of the pathogens were improved. In scarlet fever, lobar pneumonia, bacillary dysentery, Salmonella infections, and poliomyelitis the occurrence of the convalescent carrier has been well established, and no doubt these carriers play their part in the dissemination of infection. However, it seems likely that in future more attention will be given to the quantitative excretion of pathogens by the carrier and to the pathogenic potentialities of the harboured organism than to the carrier's mere existence, in assessing his importance in the spread of infection. Refinements in bacteriological techniques allow the isolation of very scanty numbers of diphtheria or dysentery or Salmonella bacilli from the throat or intestine, and such "light" carriers are often segregated in hospital until the ultimate pathogen has been eliminated, although it seems likely that they play little part in the spread of infection. On the other hand, it has been shown that the heavy nasal carrier of haemolytic streptococci is a much more dangerous source of infection than the corresponding throat carrier, apparently because he disseminates in his environment about eighty times the number of haemolytic streptococci. The convalescent intestinal carrier of poliomyelitis virus is another interesting phenomenon, for while he excretes virus in plenty he is rarely incriminated as a source of infection.

Another advance in bacteriological methods which has greatly helped in determining the source of an outbreak has been the subdivision of particular pathogens into types by antigenic analysis or by their susceptibility to specific bacteriophage. After the early work in the classification of the typhoid, Salmonella, and dysentery groups, the recognition of serological types within the genus Pneumococcus was perhaps the first step towards this subdivision of the species. Dochez and Avery in America, and independently Lister in South Africa, recognized a number of different serological types which were associated with lobar pneumonia, and this work led on to the antigenic analysis of the pneumococcus with the demonstration that serological specificity was associated with the chemical constitution of the capsular substance. As serological subdivision became extended to the pneumococci found normally in the throat as well as those associated with infection it emerged that most cases of lobar pneumonia were caused by a limited number of pneumococcal types. Thus the conception of lobar pneumonia as an infectious disease became accepted and variation in the pathogenicity of members within the species became established, although the explanation for this variation still eludes us.

The methods of antigenic analysis were extended by Bruce White and Kauffmann to the Salmonella group, by Andrewes and Inman to the dysentery bacillus, by Gordon and Murray to the meningococcus, and by Griffith and Lancefield to the haemolytic streptococci, while a biological differentiation of the diphtheria bacillus into three main types-gravis, intermedius, and mitis-was made by McLeod and his colleagues. An entirely new method of subdivision was later discovered by Craigie and Yen, who showed that a typhoid $\mathrm{Vi}$ phage could be adapted to lyse strains differentially, so that this serologically uniform organism is now classified into twenty-four phage types. This method of phage typing has been extended by Felix and Callow to paratyphoid $B$ and typhi-murium strains. The staphylococcus has also been studied intensively in recent years, and has been subdivided by both serological and phage typing, an interesting phenomenon being an apparent correlation between the two methods of typing. 
Besides its epidemiological value in tracing the source and mode of infection, this subdivision has also helped in the identification of some abnormal properties in particular members of a species. Thus certain types of Str. pyogenes, being active producers of erythrogenic toxin, are particularly associated with scarlet fever; while only two of the phage types of Staph. aureus produce enterotoxin. The different types of diphtheria bacillus differ in their pathogenicity for the human host, and variation in virulence and invasiveness has been demonstrated among the members of the Pneumococcus and Salmonella families.

\section{Modes of Spread of Infection}

The sanitary awakening of the nineteenth century, with its emphasis on the association between dirt and disease that led to the purification of water supplies and to the water-carriage system of sewerage, was followed by a rapid decline in the mortality and morbidity of the two main intestinal infections, typhoid and cholera. This campaign against environmental filth, led so vigorously by Chadwick and Simon, was based on the conception of atmospheric impurities or miasms emanating from the dunghills and privies that made many of our cities so disgusting; and environmental sanitation became the keynote of all programmes aimed at improving the health of the people. Dirt, dust, fomites, air, and water were claimed by Pettenkofer and his followers to be the main breedingplaces of the infecting germs until, early in the twentieth century, first Flügge in Germany and later Chapin in America directed attention to man as the natural reservoir of most pathogens, which must live a parasitic existence and are unlikely to survive, let alone multiply, outside the body. The poor viability of intestinal and respiratory organisms outside the body was accepted; Flügge showed that the infective particles could be sprayed to a radius of only two to three feet from a coughing tuberculous patient, and Chapin in his Sources and Modes of Infection became the champion of the direct-contact spread of infection, in opposition to the view that air, water, and inanimate objects were the important vehicles. Tetanus and anthrax were admittedly derived from soil through their resistant spores, but even these organisms did not multiply there. Typhoid was much less a water-borne infection than was generally believed, and Chapin strongly opposed the view, promulgated by Power and others, that the virus of smallpox could spread considerable distances through the air. Fomites were only rarely a means of spreading infection, and in this belief he discontinued "terminal disinfection" of houses after removal of an infectious case. Chapin's views, together with Flügge's doctrine of droplet or spray infection, led to the development of the bed-isolation and barrier technique for nursing infectious patients in fever hospitals, and the success of these methods confirmed the great importance of direct-contact infection. More recently Harries has been the chief advocate of the view that the hands play a major role in hospital cross-infection.

Rifts in the lute were soon to appear. Medical superintendents found that certain diseases like measles and chickenpox could not be nursed in barrier or cubicle wards without the risk of secondary cases, while French and German workers were proving that contaminated dust could be an important vehicle for the spread of tuberculosis. It was not, however, until the years before the 1939-45 war that direct contact as the only important mode of spread of respiratory infections began to be seriously questioned again. The work of Cruickshank, Allison, and others on the part which contaminated dust may play in the spread of streptococcal infections was followed by Wells's experiments in America demonstrating that a proportion of the droplets expelled during talking, coughing, or sneezing were so small (droplet nuclei) that they could remain suspended in the air for appreciable periods of time and be carried considerable distances from the source of infection. These findings gave fresh impetus to studies on sources and modes of spread, and led to the development of methods for controlling air-borne infection by suppression of dust (oiling of floors and fabrics) and by the use of aerial disinfectants (ultra-violet light and vaporized chemical substances). The latest development in this field is the proposed use of medicated handkerchiefs to destroy the bacteria in expelled droplets before they can be disseminated in dust particles or droplet nuclei.

As for the intestinal infections, water-borne outbreaks of typhoid fever became quite rare and most cases developed in small isolated foci where there was opportunity for spread through food and fomites. Careful search, using modern bacteriological tools, usually lead to the discovery of a chronic carrier as the source, and it is obvious that if all chronic carriers could be identified the risk of outbreaks would be greatly reduced. To this end a register of typhoid carriers in this country is now being maintained and steadily expanded at the Central Enteric Reference Laboratory. It would be advantageous if carriers could be recognized before they were incriminated as the source of an outbreak; and here again bacteriological and serological investigations of all convalescent cases will help to identify the persistent carrier. Paratyphoid fever was shown by Savage to be more closely related epidemiologically to the acute Salmonella gastro-enteritis than to typhoid fever. Both diseases are most often food-borne infections, with the highest incidence in the summer months-which indicates that a much bigger dosage of the pathogen is needed to initiate infection than is the case with Bact. typhosum. This and other evidence that these organisms and the enterotoxic staphylococci must multiply in food before causing infection has led to improved methods in the handling and storage of food.

Bacillary dysentery, which the pioneer work of Gettings at Wakefield had shown to persist in our mental hospitals largely through chronic cases and carriers, was found in the nineteen-twenties to be occurring in a mild form in the general community, and a new member of the dysentery family, the Sonne bacillus, became recognized. Since then Sonne dysentery has established itself in this country as an endemic infection, which rose to epidemic prevalence during the late war. Although explosive food-borne and, very rarely, water-borne outbreaks have occurred, its spread is usually from case to case in a manner which is still imperfectly understood, but probably occurs in very small dosage by contamination of food and fomites. With the intestinal infections, therefore, the emphasis as regards modes of spread has been changing from unsatisfactory sanitation to poor personal hygiene: routine hand-washing after use of the w.c. and before handling food would help materially in the control of these infections.

Our knowledge about the mode of spread of virus infections has until recently been based largely on epidemiological data. Although jaundice occurred as an epidemic disease in the Gallipoli campaign of 1915, the proof that catarrhal jaundice is an infectious disease (infective hepatitis) came much later and owed a great deal to the carefully collected observations of a country practitioner, W. N. Pickles, of Wensleydale, who regarded it as a droplet-spread infection with an incubation period of 
approximately four weeks, a view that has been largely corroborated from recent experience, although some observers believe there is evidence supporting an intestinal mode of spread. The American epidemiologist W. H. Frost concluded from epidemiological data that poliomyelitis was also a droplet infection, and this view is now being upheld after a period when, because the virus was found in the intestinal tract of convalescent cases, the water-borne and food-borne modes of spread were regarded as more likely.

The mode of transmission of typhus fever is a discovery of the twentieth century. Although epidemic typhus has played an important part in determining the course of history (see Zinsser's Rats, Lice, and History), it was not until 1909 that Nicolle and his colleagues showed it to be a louse-borne disease and not until 1916 that Rocha-Lima demonstrated the aetiological agent, Rickettsia prowazeki. During and after the 1914-18 war other infections-Rocky Mountain spotted fever, trench fever, and scrub-typhusbecame recognized as rickettsial infections, and in recent years two others, $Q$ fever and rickettsial pox, have been added to the list. The natural hosts and vectors (lice, rat fleas, ticks, and mites) of most of these rickettsial infections are now known, and the effective use of an insecticide in controlling the spread of epidemic typhus was well illustrated by the use of D.D.T. in the Naples outbreak at the end of the 1939-45 war.

Another pestilence about which much new knowledge has been accumulated in the past half-century is smallpox. The virus nature of the infection has been firmly established, and improved laboratory techniques-demonstration of the elementary bodies in smears, cultivation with characteristic lesions on the chick embryo, and the use of the precipitation and the complement-fixation tests-have allowed easy and early diagnosis of the atypical case. Much still remains to be discovered about the infectivity and mode of spread of smallpox, but with these new tools progress seems assured.

Hospital infection, which was such a scourge in preListerian days, again became a major problem during the 1914-18 war, so that most wounded men admitted to hospital developed secondary pyogenic infection. As a result new antiseptics like the flavines and hypochlorites were introduced, but with a return to civilian practice this problem became forgotten until the late war brought it into prominence again. An intensive attack, led by the bacteriologists armed with new methods for tracing the spread of the haemolytic streptococcus and other pathogens, resulted in great improvements, such as the "no-touch" technique, in methods for dealing with wound infections. Towards the end of the, war penicillin, used on a large scale prophylactically and therapeutically, achieved remarkable results in controlling both gas gangrene and the pyogenic infections.

\section{Protection of Susceptibles}

The system of dealing with infectious disease by notifcation, isolation, and quarantine, initiated in the last century and developed in the early decades of the present, was designed partly to afford suitable treatment for the patient and partly to reduce the risk of infection among contacts. Although sometimes a life-saving measure, as in diphtheria. hospital treatment of infections like measles and scarlet fever frequently failed to achieve its main purposes, for the patient developed secondary complications from crossinfection after admission, and often managed to infect susceptible contacts before his removal or after his return home. Although mortality rates of most infectious diseases have shown remarkable, if uneven, reduction in the past half-century, the incidence of the respiratory group of diseases has changed very little, and as many children develop measles and whooping-cough to-day as fifty years ago. Obviously some more specific means of protecting susceptibles was needed.

Although typhoid fever in the civilian population was quickly coming under control with improved sanitation, the ravages from this disease during wars was an important stimulus to the discovery of more effective preventive measures. Almroth Wright had gone to be professor of pathology at the Army Medical School at Netley in 1892, and from 1895 onwards he developed his conception of active immunization against typhoid fever by the use of killed vaccines, which was a "break-away" from the Pasteurian practice of using attenuated living cultures. He standardized the dosage and, having satisfied himself that inoculations of killed vaccine rendered the blood highly bactericidal to the typhoid bacillus, he urged the adoption of active immunization for our armies overseas. His proposal was at first rejected by the War Office, but a Royal Commission reversed this decision, and antityphoid inoculation was adopted as a standard procedure for the British Army. The benefit was not fully reaped till the 1914-18 war, when the annual incidence and death rate from typhoid per thousand strength were respectively 2.35 and 0.139 , compared with figures of 105 and 14.6 in the South African War. Later, Arkwright's classical studies on the change in Salmonella bacilli during artificial culture from the smooth (virulent) to the rough (avirulent) variant led to the preparation of typhoid vaccines from smooth mouse-virulent strains, and after Felix had demonstrated the importance of a heatlabile antigen (the virulence or $\mathrm{Vi}$ antigen) alcohol-killed and alcohol-preserved vaccine was introduced as a further improvement.

Meanwhile, although progress had been made in the preparation and use of diphtheria antitoxin, so that case mortality was reduced to about one-third of that of the pre-antitoxin era, deaths from diphtheria remained at figures around 2,500 and 3,000 , with an incidence of 50,000 to 60,000 , per annum. It was known that susceptible animals could be immunized against diphtheritic infection, but early attempts to immunize man by using small doses of toxin or bacterial vaccine gave little promise of success. Theobald Smith's observations that suitable mixtures of toxin and antitoxin. could be used as immunizing agents were not followed up until 1913, when Schick described a skin test for differentiating susceptible from immune persons and von Behring reported that toxin-antitoxin mixtures could safely be injected into children. W. H. Park, of New York, then became the leader in the campaign for the immunization of children against diphtheria, and his energy and enthusiasm were in large part responsible for the progress made in America at a time when, despite advances in the laboratory, Britain was still holding back. The leader in the laboratory was A. T. Glenny, who produced first diphtheria toxoid (toxin rendered innocuous but still highly antigenic by the addition of formalin), then the toxoidantitoxin mixture (T.A.M.), then toxoid-antitoxin floccules (T.A.F.), a remarkably safe and efficient prophylactic, and lastly alum-precipitated toxoid (A.P.T.), which was shown to have greatly increased antigenic activity associated with its slow absorption and excretion. The latest development in diphtheria prophylaxis is the preparation by Holt of purified toxoid precipitated in optimum proportion with aluminium phosphate (P.T.A.P.) and constituting an antigen which is remarkably uniform, efficient, and safe in its action. 
Although Britain was a late starter in large-scale immunization against diphtheria, the national campaign, begun in 1940 with A.P.T. as the antigen of choice, has in the past eight years achieved phenomenal results. Some 60-70\% of school and pre-school children have been immunized, and notifications of diphtheria have fallen from an average of 60,000 in pre-war years to 3,531 in 1948 , with only 156 deaths.

Wars often act as accelerators to the advance of medical science. Protection of the wounded against tetanus was first attempted on a large scale during the 1914-18 war by prophylactic injection of tetanus antitoxin at the time of wounding. Later the demonstration that both horses and men could be effectively immunized with tetanus toxoid led to the adoption of active immunization during the 1939-45 war, supplemented, in the British Forces, by passive immunization at the time of wounding. As a result the incidence of tetanus fell from rates of $15-30$ per 1,000 wounded in the early stages of the 1914-18 war to less than 1 per 1,000 when tetanus antitoxin became generally used, and to rates that varied from 0.04 (B.N.A.F.) and 0.06 (B.L.A.) to 0.43 (B.E.F.) per 1,000 wounded in the different campaigns of the 1939-45 war. Knowledge about the aetiology and means of control of gas gangrene developed from studies in the 1914-18 war, when the pathology and bacteriology of this virulent infection was established, and the first controlled trial in specific prophylaxis was carried out with Clostridium welchii antitoxin. The value of gasgangrene antitoxins in the prophylaxis and treatment of this composite infection is still a matter for discussion, but here again there is promise of progress in the use of toxoid for active immunization. The stimulus of war also led to rapid developments in the preparation of vaccines for protection against typhus and other rickettsial infections. Apart from living vaccines prepared in animal tissues, the only killed vaccine in use against typhus before the 1939-45 war was the Weigl vaccine derived from lice after rectal injection. The cultivation of the rickettsia in chick embryo resulted in the large-scale production of egg-yolk vaccine, which was used with remarkable success to protect the Allied troops fighting in areas where typhus was endemic.

Between the wars the field of viruses was being tilled with vigour and was beginning to yield fruit. At the National Institute for Medical Research, which had been established by the Medical Research Council to carry out organized research on biological problems, the work of Laidlaw and Dunkin on dog distemper, with the ferret as experimental animal, resulted in a practical method of immunization, and probably stimulated the fresh attack on the aetiology of influenza which led to the discovery of a specific virus by the Hampstead team. The adaptation of this virus, first isolated in the ferret, to the mouse and the chick embryo allowed its production on a sufficient scale for the preparation of vaccines, which have been used with some success in the prophylaxis of influenza. It is now known, however, that besides two main serological types of influenza virus called $\mathbf{A}$ and $\mathbf{B}$ there are antigenic variants within each of these types, so that a vaccine prepared with a particular A strain máy not protect against another member of the family.

The demonstration that certain fractions of an organism -for example, the $O$ antigen of the Salmonella group and the capsular polysaccharide of the pneumococcus-are particularly associated with pathogenicity encouraged the immuno-chemists to produce pure preparations of these fractions for use as immunizing agents. Although some success has been obtained experimentally with $O$ fractions of Salmonella and Shiga dysentery bacilli, the most promising bacterial fraction has been the specific pneumococcus polysaccharides, which when injected in infinitesimally small amounts have proved to be protective against infection with the corresponding pneumococcus type. Progress has also been made in the combination of two or more antigens-for example, in immunization against typhoid and tetanus or against diphtheria, pertussis, and tetanus. Such combined prophylactics do not interfere with the antigenicity of each component, but seem rather to encourage better antibody production than when the antigens are used singly, and have the administrative advantage of reducing the number of injections. Combined active and passive immunization has been found to be a practicable and useful procedure against both diphtheria and tetanus.

The recent use of living vaccines represents another turn of the wheel. Lymph vaccine has, of course, long been used to protect against smallpox, but the development of a live yellow fever vaccine has proved to be perhaps the only artificial method of immunization that gives complete protection. Live vaccines of Brucella abortus and distemper virus have come into effective use in veterinary practice, and the B.C.G. tubercle vaccine is establishing itself in many countries as one method of reducing morbidity and mortality from tuberculosis.

\section{Treatment of Infection}

The successful production and therapeutic use of diphtheria antitoxin before the end of the nineteenth century were followed by much less successful attempts to treat other infections, from tetanus to typhoid, with specific antisera. Where disease symptoms were due to powerful exotoxins, it became apparent that these toxins were selectively fixed to tissue cells so quickly and firmly that the specific antisera could have only a prophylactic effect. This has proved to be the case with tetanus and to a large extent with diphtheria, so that it is only in cases treated in the first few days of infection that antitoxin is beneficial. Antibacterial sera had not achieved much success until antipneumococcal sera, concentrated by a new process by Felton, yielded such promising results as to encourage large-scale production of different type-specific antisera, first in horses and later in rabbits. However, just as this method of treating lobar pneumonia was being adopted by other countries than America, where it was first introduced, Whitby, in 1938, showed that one of the new sulphonamide drugs, sulphapyridine ("M and B 693 "), was effective against pneumococcal infections, and chemotherapy quickly supplanted serotherapy. Within the next two years the sulphonamides had perhaps their greatest triumph against the largest epidemic of cerebrospinal meningitis that this country has ever experienced.

This extension of chemotherapy to infections other than the spirochaetal diseases had to wait a quarter of a century after Ehrlich's discovery of "salvarsan," and was thought at first to be another example of the specific affinity of a dye-like antiseptic for the bacterial cell. However, the researches of Fildes and Woods on bacterial metabolism indicated that the chemotherapeutic action of these new drugs depended on competitive inhibition by the sulphonamides against an essential metabolite, in this case paraamino-benzoic acid. Other analogous types of inhibition were shown to occur, and this new approach has opened the way to a rational attack on the bacterial cell. Meanwhile, in America Dubos was attempting to isolate antibiotic substances by using killed cultures of staphylococci, 
pneumococci, and streptococci as the substrate for soil bacteria, and his success with tyrothricin and gramicidin was probably the stimulus which led Florey and Chain to investigate penicillin, a mould extract whose properties and potentialities as a chemotherapeutic agent had been described by Fleming ten years earlier. In the past few years many thousands of antibiotics have been isolated, but only a few have been found to be therapeutically useful. However, armed with the sulphonamides, penicillin, and streptomycin, and soon, we hope, with chloramphenicol and aureomycin, the medical practitioner can to-day tackle most bacterial infections with reasonable hopes of success. The therapeutic activity of the latter two drugs against the rickettsia and some of the larger viruses gives promise of still further successes.

Besides their clinical value in the treatment of established infection, the new drugs are by their bactericidal and bacteriostatic activity helping to eliminate the reservoirs of infection, particularly in convalescent and contact carriers. This effect was demonstrated in the use of sulphonamides for the treatment of bacillary dysentery, since the drug secured early bacteriological as well as clinical cure, and this action probably helped to curtail the spread of dysentery among our troops during the 1939-45 war. The sulphonamides have been equally effective in eliminating the meningococcus and gonococcus, and have been used prophylactically to control the spread of infection due to these and other organisms and to prevent relapses of rheumatic fever. Penicillin has also been used successfully to eliminate the haemolytic streptococcus and diphtheria bacillus from the upper respiratory tract of acute cases and carriers. A new danger, however, is the risk of the development of drug-resistant bacteria, exemplified by the sulphonamide resistant gonococcus and haemolytic streptococcus, the penicillin-resistant staphylococcus, and the streptomycin-resistant tubercle bacillus. So far, no satisfactory solution of this problem has been forthcoming.

\section{Summing Up}

With bacteriology still in its first vigour around 1900, it was natural that the parasite should be regarded as the most important factor in the occurrence of infection. The success of vaccine prophylaxis and of chemotherapy helped to establish the bacteriologist. as an equal with his clinical colleagues, and for a time this new branch of medicine flourished better than perhaps it deserved. However, the development of immunology and the widening interest in bacteriology as a science in itself gradually led to the conception of infection as a struggle for existence between the parasite and its host. This oecological approach finds support in the history of infectious diseases, which after the first violent interaction, as when syphilis invaded the Old World, tend to reach an approximately balanced relationship between host and parasite, so that the survival of each is assured. For the parasite the main problem has become an easy means of transfer from one host to another. Some of the vehicles, like water and milk, have been made particularly hazardous, and intestinal infections have correspondingly declined. Blockage of the channels of spread of respiratory infections presents much more difficult problems; but dust is already being recognized as an important and controllable vehicle, and clean air in intramural spaces may some day become as commonplace as clean milk and water.

This attack on the parasite by improvements in environmental sanitation has been accompanied on the one hand by new knowledge of the reservoirs of infection and the means for their eradication, and, on the other, by successful efforts at raising the resistance of the host by specific and non-specific means. The history of diphtheria shows that no measure short of specific immunization made any progress toward its eradication, and this would seem to be the method of attack with infections like whooping-cough and measles. But history warns us again that there is no single golden rule for the control of infectious diseases, and each infection must be studied as an entity. Improvements in social conditions, and particularly in the nutrition of the community, have greatly helped in reducing mortality in most infectious diseases, but again there is no room for complacency, for the death rates from measles and whooping-cough are still many times higher among the poor than among the rich.

The following general literature is suggested for the interested reader :

C. V. Chapin, Sources and Modes of Infection. New York, 1910.

J. C. G. Ledingham, and J. A. Arkwright, The Carrier Problem in Infectious Diseases. London, 1912.

W. W. C. Topley, An Outline of Immunity. London, 1933. H. H. Scott, Some Notable Epidemics. London, 1934.

Theobald Smith, Parasitism and Disease. London, 1934. Major Greenwood, Epidemics and Crowd Diseases. Harvard University Press, 1935.

W. N. Pickles, Epidemiology in Country Practice. Bristol, 1939.

F. M. Burnet, Biological Aspects of Infectious Disease. Cambridge, 1940.

C.-E. A. Winslow, The Conquest of Epidemic Disease. London, 1943.

R. J. Dubos, The Bacterial Cell. London, 1944.

\section{THE DEVELOPMENT OF PSYCHOLOGICAL MEDICINE}

BY

\section{JQHN RICKMAN, M.D. \\ President of the Institute of Psycho-analysis}

The half-century which this series of essays is designed to cover also spans the history of modern psychological medicine, which is also the history of a new skill. Every advance in medical science can be related to the development of skills -in clinical observation, in the design and use of apparatus of research, in the interpretation of data obtained by the use of the apparatus, and above all in conceptualizing the problem that is being faced. To this general rule the development of psychological medicine is no exception; but since the data differ so greatly from those of the physiologist and pathologist the means by which the facts are collected also are different. Both kinds of research are concerned with answers to the fundamental question, "What goes with what?" If the data of the pathologist cannot be immediately linked with the data of the psychopathologist and psychiatrist, and vice versa, it simply means that "total medicine" has not yet found a theory which combines the two.

This article does not touch on the development of psychiatry but tries only to outline some of the changes in thought and practice that have resulted from the study of the psychological problems which patients present to doctors for solution. To summarize in a short article the changes in the last fifty years would exhaust the general reader and weary the specialist, but a description of how it all arose from simple if subtle consulting-room work (without appliances of any kind whatever) and how the range of case-taking has been extended in penetration and 